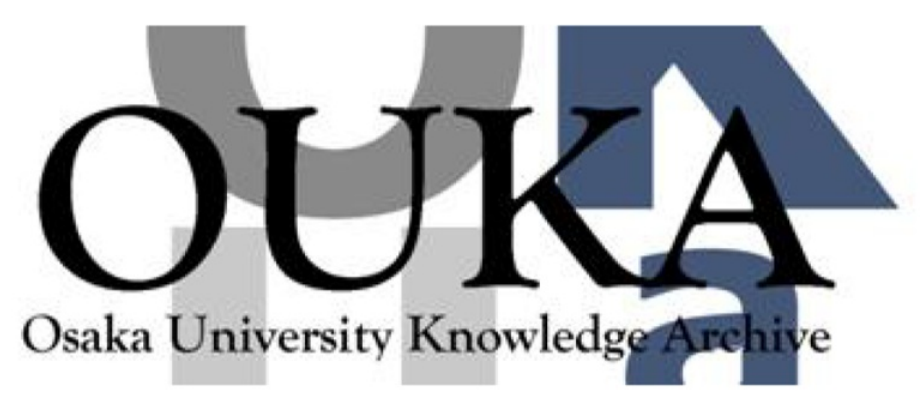

\begin{tabular}{|c|c|}
\hline Title & $\begin{array}{l}\text { Toward Vision-Based Intelligent Navigator: Its } \\
\text { Concept and Prototype }\end{array}$ \\
\hline Author (s) & Miura, Jun; Itoh, Motokuni; Shirai, Yoshiaki \\
\hline Citation & $\begin{array}{l}\text { IEEE Transactions on Intelligent Transportation } \\
\text { Systems. } 3(2) \text { p. } 136-\text { p. } 146\end{array}$ \\
\hline Issue Date & $2002-06$ \\
\hline oaire:version & VoR \\
\hline URL & https://hdl. handle. net/11094/3363 \\
\hline rights & $\begin{array}{l}\text { ๑2002 IEEE. Personal use of this material is } \\
\text { permitted. However, permission to } \\
\text { reprint/republish this material for advertising } \\
\text { or promotional purposes or for creating new } \\
\text { collective works for resale or redistribution } \\
\text { to servers or lists, or to reuse any } \\
\text { copyrighted component of this work in other } \\
\text { works must be obtained from the IEEE.. }\end{array}$ \\
\hline Note & \\
\hline
\end{tabular}

Osaka University Knowledge Archive : OUKA

https://ir. Library. osaka-u. ac. jp/

Osaka University 


\title{
Toward Vision-Based Intelligent Navigator: Its Concept and Prototype
}

\author{
Jun Miura, Member, IEEE, Motokuni Itoh, and Yoshiaki Shirai
}

\begin{abstract}
This paper proposes a novel concept of intelligent navigator that can give the driver timely advice on safe and efficient driving. From both the current traffic condition obtained from visual data and the driver's goal and preference in driving, it autonomously generates advice and gives it to the driver. Not only operational level advice, such as emergency braking due to an abrupt deceleration of the front vehicle, but also tactical-level advice, such as lane changing due to the congested situation ahead, can be generated. Two main components of the intelligent navigator, the advice generation system and the road scene recognition system, are explained. A three-level reasoning architecture is proposed for generating advice in dynamic and uncertain traffic environments. Online experiments using the prototype system show the potential feasibility of the proposed concept.
\end{abstract}

Index Terms-Hierarchical reasoning architecture, intelligent navigator, intelligent transportation systems (ITS), visual recognition of traffic scene.

\section{INTRODUCTION}

I $\mathrm{N}$ RECENT years, there has been growing interest in intelligent transportation systems (ITS). One ultimate goal of ITS research is to realize a fully autonomous vehicle [1], [2]. It is, however, still difficult to achieve this goal because very high reliability and safety will be required for deployment. Thus, as a practical step toward the goal, we propose the intelligent navigator, that can, in place of a human navigator sitting on the next seat, give the driver appropriate advice on safe and efficient driving.

To drive to a destination, we first select a route from the current location to the destination, and then drive a vehicle to follow the route. Concerning the assistance to route selection, navigation systems have already been deployed which use various information such as GPS data, map data, and traffic condition information provided by local traffic control centers [3]. On the other hand, the assistance to driving itself is still an active research area. This is mainly due to lack of reliable sensory systems and intelligent assistance planner.

Tasks in driving can usually be divided into two levels: at the higher level, maneuvers such as lane changing and overtaking are determined to meet the objective of driving (e.g., a

Manuscript received November 13, 1999; revised April 15, 2002. The Associate Editor for this paper was S. Washino.

J. Miura and Y. Shirai are with the Department of Computer-Controlled Mechanical Systems, Graduate School of Engineering, Osaka University, Osaka 565-0871, Japan (e-mail: jun.miura@ieee.org; shirai@mech.eng.osaka-u.ac.jp).

M. Itoh is with the Graduate School of Science and Technology, Kobe University, Kobe 657-8501, Japan.

Publisher Item Identifier 10.1109/TITS.2002.801421. target arrival time) under the constraints imposed by the actual traffic condition; at the lower level, the selected maneuver is translated into actual operations of steering, accelerating, and braking. These levels are called tactical level and the operational level, respectively [4], [5].

Operational level driving can be assisted relatively straightforwardly using various sensing capabilities such as vision for lane detection [6] or for detecting other vehicles [7], [8]; if some dangerous situation arises, the driver can be warned. Several commercial products for local level assistance have recently been reported [9]. However, so far, there has been minimal development on assistance systems for the tactical level.

Sukthankar et al. [5] pointed out the importance of tacticallevel driving in realizing safe and efficient autonomous driving. This is also true for driver assistance systems. Since the quality of maneuver selection may have considerable effects on safety and efficiency, it is important to generate advice on appropriate maneuvers (i.e., tactical-level advice) in a timely fashion.

In this paper, we propose an architecture of intelligent navigator which can generate tactical-level advice as well as local level advice. Fig. 1 schematically depicts the proposed architecture of the intelligent navigator system. The driver gives the system the goal of driving (e.g., the target arrival time) and his/her preference to specific driving styles (e.g., the driver may want to avoid lane changing as much as possible). The road scene recognition subsystem recognizes the current traffic situation using vision. The advice-generation subsystem generates appropriate advice and give it to the driver. The driver may control the vehicle according to the given advice. Since there have been many works on visual recognition of road scenes, we can adopt their results to implement our road scene recognition system. Concerning generation of tactical-level advice, however, there has been research done previously; therefore, this paper focuses on how to construct such an advice generation system.

In designing an advice generation system, we have to consider the following two issues. One is the uncertainty (or ambiguity) in road scene recognition results based on which advice is generated. The other is the dynamics of road scene; i.e., the traffic situation evolves as time advances. Therefore, the tactical-level advice should be generated based on the prediction of the future traffic condition with consideration of uncertainty. As a mechanism for such advice generation, we propose a three-level reasoning architecture with probabilistic traffic modeling.

We have developed an intelligent navigator prototype by combining the advice generation system, which is based on the layered reasoning architecture, with a vision system to detect lanes and vehicles. The prototype is for driving on a highway 


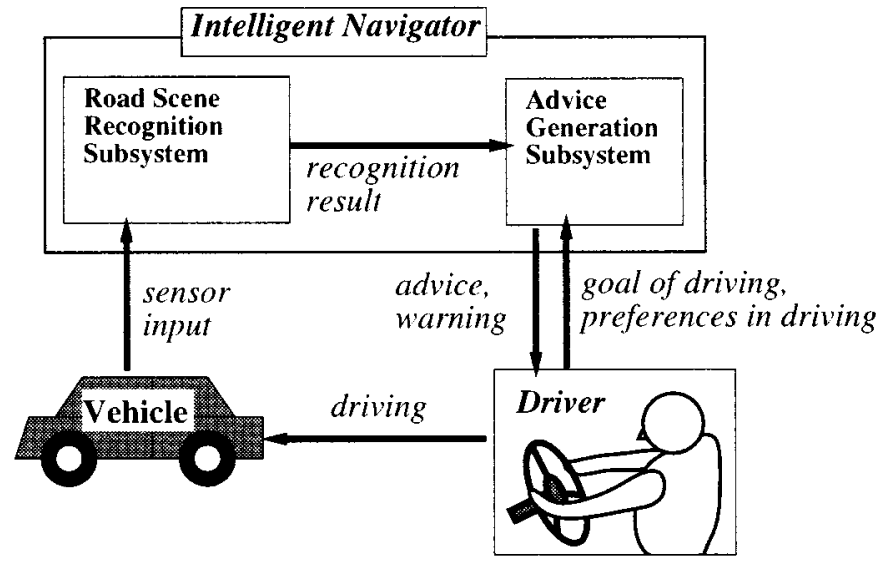

Fig. 1. Overview of intelligent navigator system.

without branches and is implemented as a stand-alone system such that it is easily installed in a normal, unmodified vehicle. To the authors' knowledge, the prototype is the first online system for tactical-level advice generation.

It is very costly to always use a real prototype for verifying the advice-generation algorithm and the traffic models, therefore, we developed a highway traffic simulator. Before online experiments, we ran numerous simulated experiments to improve the algorithms and the models. The simulator can generate various traffic conditions by setting the road configuration, the number of vehicles, and the driving strategy of each vehicle.

The rest of the paper is organized as follows. Section II describes the proposed three-level reasoning architecture with comparison with previous works. Section III explains how to generate tactical-level advice; that is, how the driver gives the system the goal of driving and his/her preferences in driving, how the uncertain and dynamic traffic situation is modeled probabilistically, and how tactical-level advice is generated from such information. The metatactical-level reasoning is also explained in this section. Section IV describes our simple but robust traffic scene recognition subsystem. Section V describes preliminary experimental results. Section VI summarizes the paper and discusses future works.

\section{ThreE-LeVEl REASONING ARCHITECTURE}

This section describes our three-level reasoning architecture suitable for advice generation in uncertain and dynamic environments.

There have been several works on decision-making considering the uncertainty and the dynamics of environments. Niehaus and Stengel [10] modeled the movement of a nearby vehicle using a probabilistic distribution, which is continuously updated using the Kalman filtering, and generated a safe plan considering the probable worst-case scenarios. Only a local and short-time prediction is performed in planning. Forbes et al. [11] proposed to model all levels of planning for an automated vehicle using a fixed probabilistic network. Although they proposed an efficient computation method for the network, extending the approach to more complicated scenarios may still be difficult because of increasing computational cost. Sukthankar et al. [5] proposed a distributed reasoning scheme

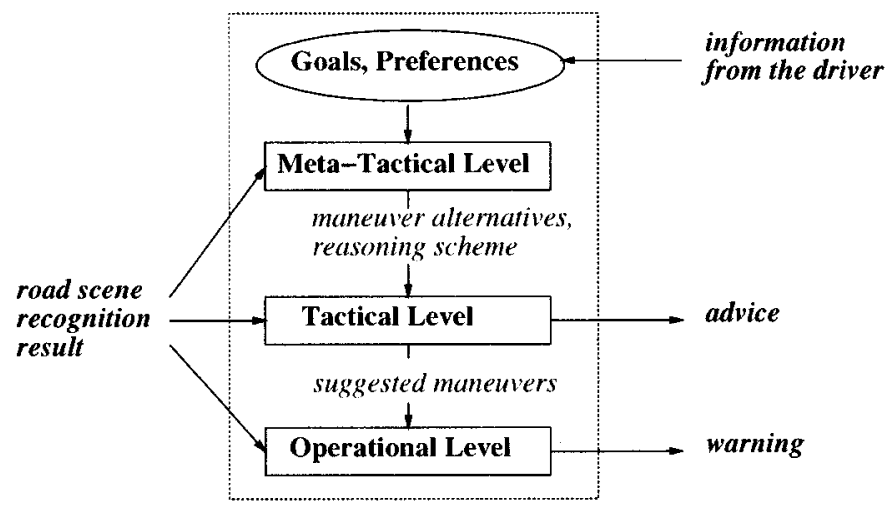

Fig. 2. Three-level architecture of advice-generation subsystem.

for the tactical-level planning. Independently operating planning modules with different algorithms vote for the desirable action, and the high-scored action is selected and executed. The parameters and the relative weight of each planning module are tuned through an evolutionary learning method. The proposed scheme seems fitted to the tactical-level planning that requires a relatively short-term prediction.

To make a plan with a long look-ahead tends to be computationally expensive if all alternatives are considered in every situation. Moreover, it may be inefficient to always carry out such a planning. Therefore, we propose to introduce a knowledge-based metalevel planning (called the metatactical level) to control the tactical level, i.e., to adaptively limit the search space of the tactical level and to activate the tactical level only when it is necessary, according to both the history of maneuvers and the current traffic condition. The resultant control architecture is composed of three levels: metatactical level, tactical level, and operational level.

Fig. 2 illustrates the internal architecture of the advice-generation subsystem, which is based on the above three-level reasoning architecture. The metatactical level continuously watches designated events on traffic and, on occurrence of an event, activates an appropriate tactical-level maneuver selection procedure. Then the tactical level determines the best maneuver to suggest and give it to the driver. The operational level mainly checks immediate dangers, such as an abrupt deceleration of the front vehicle, by watching near surrounding areas of the vehicle.

In robotics, several layered architectures have been proposed. Gat [12] proposed a three-level control architecture for autonomous robots. In his architecture, called ATLANTIS, the controller is responsible for controlling primitive activities, which are usually reactive sensorimotor processes; the deliberator controls time-consuming computational activities such as planning and world model maintenance; the sequencer coordinates such various activities by initiating and terminating them according to the current goal and situation. Pell et al. [13] proposed a similar architecture for autonomous spacecraft. Such works mainly discuss how to integrate deliberative and reactive activities, which correspond to the tactical- and operational levels of our architecture, respectively. Therefore, our approach of putting a knowledge-based metalevel is different from those of the previous works; the metalevel can also be applied to their architectures. 


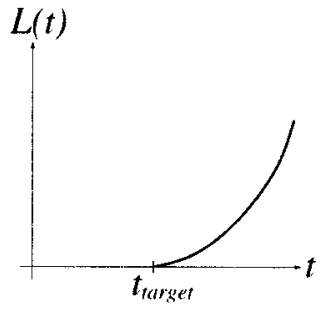

(a)

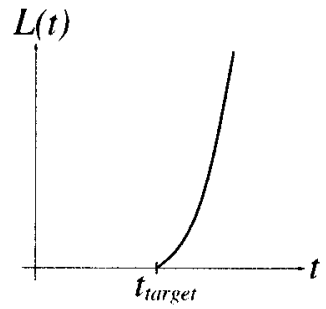

(b)

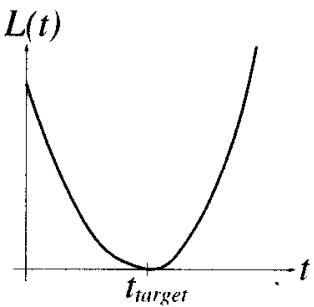

(c)

Fig. 3. Examples of $L(t)$ : (a) loose requirement on the arrival time, (b) tight requirement on the arrival time, (c) both late and early arrivals are undesirable.

\section{Generating TACTiCAl LeVel AdVice}

\section{A. Information from the Driver}

The intelligent navigator receives the driver's goal and preference in driving which are to be used for advice generation. We represent such information in the forms of loss function and cost assignment, because a statistical decision theory is used for advice generation.

Loss function $L(t)$ is used to represent drivers' requirements on the time of arrival at the destination (the target exit). $L(t)$ is defined using the target arrival time $t_{\text {target }}$ and the estimated arrival time $t$. Fig. 3 shows some examples of $L(t)$. A tighter requirement on $t_{\text {target }}$ is represented by a larger gradient of $L(t)$. The loss function could be changed during driving according to the change of the goal.

Cost $C$ is used to represent the driver's degree of preference to each maneuver. For example, $C_{\text {change }}$ is the cost related to the lane-changing maneuver; if the driver puts the largest importance on safety and very much wants to avoid possible risks related to lane changing, a high $C_{\text {change }}$ is given to the system so that the lane-changing maneuver is least likely selected except in a truly emergency situation.

\section{B. Tactical-Level Reasoning Considering Uncertainty and Ambiguity of Road Scene Recognition}

The tactical-level reasoning basically runs as follows. First, the expectation of the arrival time at the destination when each maneuver is adopted is estimated from the probabilistic model of the current traffic situation. Using the estimated arrival time, we calculate the loss of each candidate maneuver using the loss function and the cost attached to the maneuver. Finally the best maneuver is selected which minimizes the loss.

We also consider the ambiguity of a recognition result. When the road is moderately occupied by vehicles, the visual recognition subsystem attached to the vehicle cannot obtain information for the whole surroundings due to occlusion and, thus, may not be able to determine the traffic situation uniquely. In such a case, the best maneuver in each possible situation is first selected, and then, if a maneuver is selected in every situation, that one is selected for advice. Otherwise, any advice is not generated. Using information from the driver, however, a useful advice could be generated. That is, since the recognition ability of the driver is usually better than that of the recognition system, the driver may have more information (e.g., the driver can see the vehicles far ahead through the windows of the front vehicle). Therefore, the system provides conditional advice which complimentarily uses

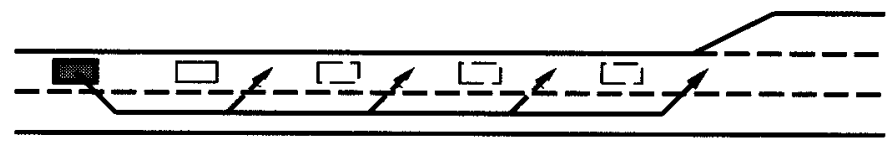

(a)

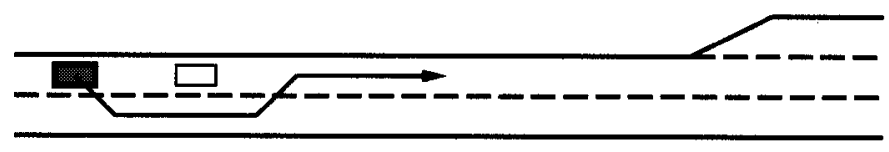

(b)

Fig. 4. Estimated traffic situations and overtaking scenario. (a) Vehicles are almost equally placed in the lane. (b) Only a few (slow) vehicles are in the lane.

the driver's recognition ability, when it is useful. An example is shown below.

Consider the scenario shown in Fig. $4 .{ }^{1}$ The vehicle with the intelligent navigator (called MyVehicle, drawn as a painted rectangle in the figure) on the left lane ${ }^{2}$ is approaching its exit. Since the speed in the current lane is becoming a little bit slow, the intelligent navigator starts thinking of advising the driver to overtake vehicles ahead. The overtaking maneuver is generally faster, but there may be risks of lane changing itself and of missing the exit. We will evaluate two maneuvers, keep-lane and overtake. Suppose only vehicles just before and behind MyVehicle are visible. There are two possible cases for the occluded area:

- congested: vehicles are almost equally placed in the lane [see Fig. 4(a)];

- not congested: just a few (slow) vehicles are blocking our lane [see Fig. 4(b)].

The system tries to identify the situation using the method described in Section III-C. If the situation is identified, we evaluate candidate maneuvers for the identified situation, otherwise, the evaluation is carried out for both situations.

Let $t_{A}$ be the estimated arrival time of overtake maneuver in the congested situation, $t_{B}$ be that of the same maneuver in the other situation, and $t_{S}$ be that of keep-lane maneuver (the result of this maneuver is supposed to be equal in both situation). In addition, the cost $C=C_{\text {change }}$ is considered for overtake maneuver.

The result of comparison and given advice are as follows:

- if $L\left(t_{A}\right)+C>L\left(t_{B}\right)+C>L\left(t_{S}\right)$, keep-lane maneuver is always better than the other; the given advice is "keep lane" [see Fig. 5(a)];

${ }^{1}$ This was originally presented by Sukthankar [5].

${ }^{2}$ Note that the slower lane is the left one in Japan. 


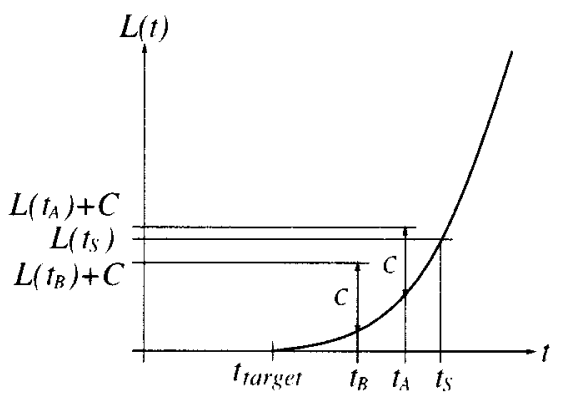

(a)

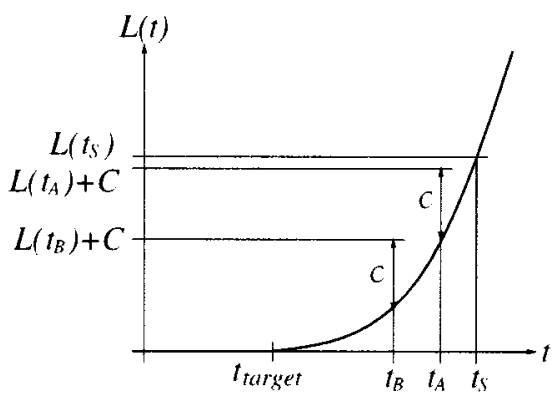

(b)

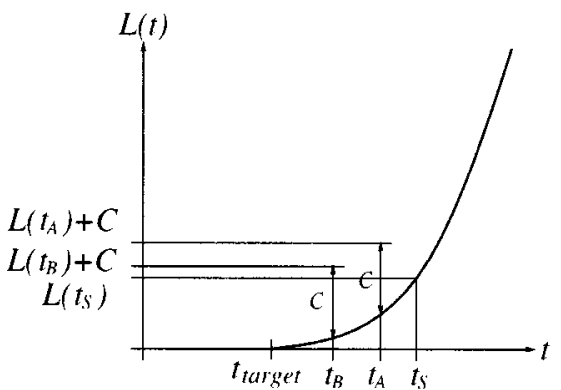

(c)

Fig. 5. Maneuver evaluation and given advice. (a) "Keep lane." (b) "Change lane." (c) "Change lane" if not congested.

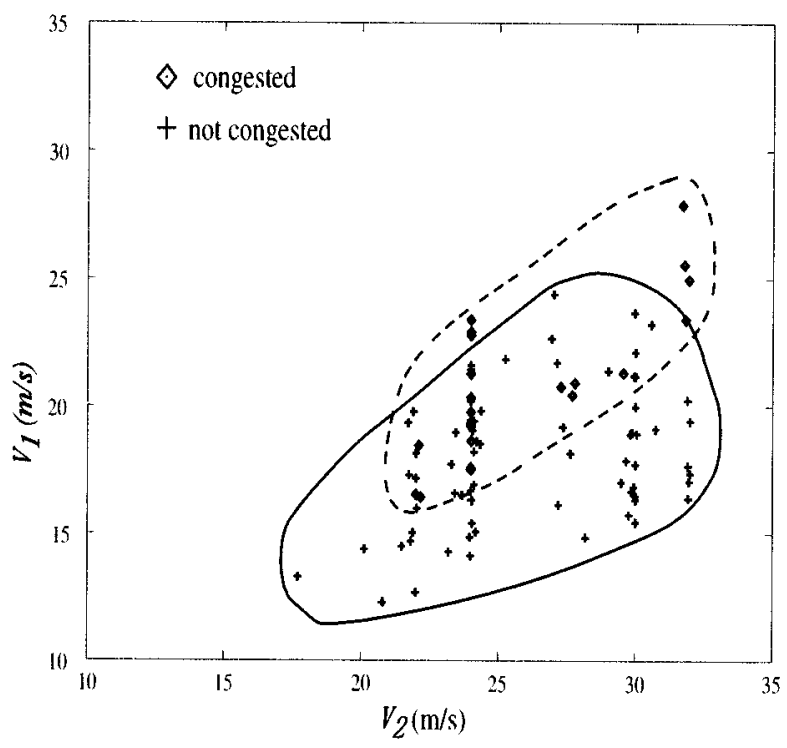

Fig. 6. Result of simulation experiment for the velocity map.

- if $L\left(t_{S}\right)>L\left(t_{A}\right)+C>L\left(t_{B}\right)+C$, overtake maneuver is always better; the advice is "change lane" [see Fig. 5(b)];

- Otherwise (i.e., $L\left(t_{A}\right)+C>L\left(t_{S}\right)>L\left(t_{B}\right)+C$ ), overtake maneuver is better only in not congested situation; thus, the conditional advice "change lane if not congested" is generated [see Fig. 5(c)].

\section{Traffic Situation Estimation Using Velocity Map}

In some cases, it is possible to estimate the situation of occluded areas by the velocity difference of the two lanes. For example, normally the velocity difference can be supposed to be small at the position far from any entrance or exit. If the velocity of our lane is slower than the other, it is likely to be that just a few slow vehicles are blocking our lane and it is not congested.

We verified the validity of the state estimation using the highway-traffic simulator. We performed numerous simulations assigning various parameters to the vehicles; in each situation that occurred, we recorded the average velocity of both lanes and, at the same time, manually classified the situation into congested and not congested. Fig. 6 shows the compiled result for the case that the distance to the next exit is medium. In the figure, $v_{1}$ and $v_{2}$ represent the average velocity in the

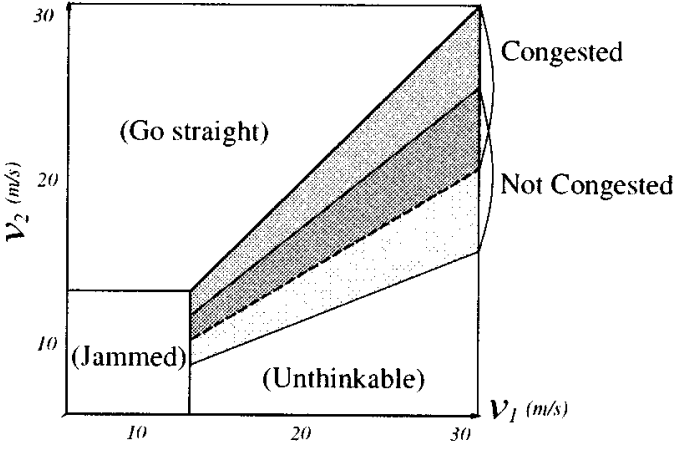

Fig. 7. Example of the velocity map.

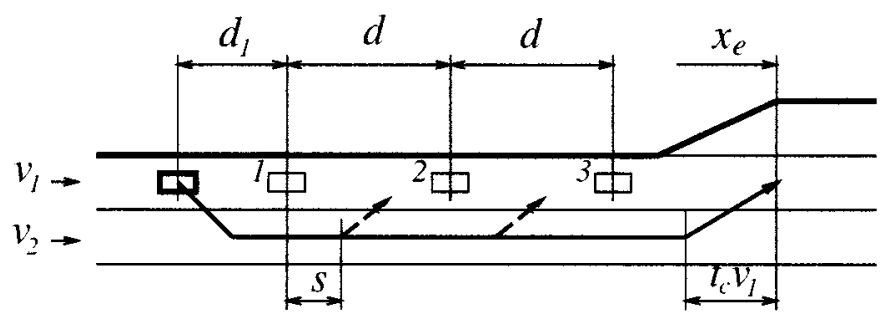

Fig. 8. Overtaking with consideration of approaching exit.

slower and the fast lanes, respectively. The result shows that the congested situation occurs when the velocity difference is smaller (the area in the dotted line) and the not congested situation occurs when the velocity difference is large (the area in the solid line). Using the result, we constructed the velocity map like the one shown in Fig. 7 that supports the estimation of occluded areas.

\section{Example of Probabilistic Traffic Modeling: Overtaking With Consideration of Approaching Exit}

This subsection describes an example of probabilistic traffic modeling. The situation for modeling is the one shown in Fig. 4(a). Using the model, we calculate the expectation of the arrival time at the target exit when taking overtake maneuver (i.e., overtaking front vehicles and returning to the left lane before the exit).

Let us consider Fig. 8. Let $v_{1}$ and $v_{2}$ be the average speed on the left and the right lanes, respectively, and $d$ be the distance between vehicles in the occluded areas. We model the uncertainty of $d$ by a normal distribution. Let $\mu$ and $\sigma^{2}$ be the mean and the 


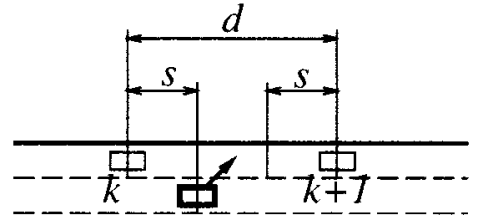

(a)

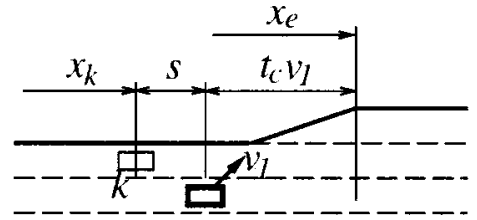

(b)

Fig. 9. Conditions for overtaking. (a) Condition for successful cut-in. (b) Condition for successful exiting.

variance of the normal distribution, respectively. These parameters are related to the lane speed, and have been empirically obtained [14]. Given $\mu$ and $\sigma^{2}$, the distribution of the current position $x_{k}(0)$ of the $k$ th $\operatorname{car}(k \geq 2)$ ahead at time $t=0$ is specified by the following mean $u_{k}$ and variance $\sigma_{k}^{2}$ :

$$
\begin{aligned}
& \mu_{k}=d_{1}+(k-1) \mu \\
& \sigma_{k}^{2}=\sigma_{1}^{2}+(k-1) \sigma^{2}
\end{aligned}
$$

where $d_{1}$ and $\sigma_{1}^{2}$ are the mean and the variance of the distance to the front vehicle, respectively, obtained by vision.

Let us calculate the probability that MyVehicle can safely takes the exit after overtaking $k$ cars ahead. First, we consider the condition that MyVehicle can cut in the space between the $k$ th and $k+1$ th vehicles [see Fig. 9(a)]. The probability $P_{i_{k}}$ that this condition holds is given by

$$
P_{i_{k}}=P(2 s \leq d)
$$

where $s$ is the safety margin for entering. Next, consider the condition that MyVehicle does not miss the exit. This condition is restated as the condition that the position of MyVehicle when it finishes overtaking the $k$ th vehicle is sufficiently before the exit [see Fig. 9(b)]. The probability that this condition holds is calculated as

$$
P_{e_{k}}=P\left(x_{k}\left(t_{k}\right)+s \leq x_{e}-t_{c} v_{1}\right)
$$

where $t_{c}$ is the time for lane changing, $x_{e}$ is the position of the exit; $t_{k}$ is the time for overtaking $k$ vehicles. Since $t_{k}$ satisfies the following equation:

$$
x_{k}\left(t_{k}\right)=x_{k}(0)+v_{1} t_{k}=v_{2} t_{k}-s
$$

$t_{k}$ is given by

$$
t_{k}=\frac{x_{k}(0)+s}{v_{2}-v_{1}} .
$$

From (4)-(6), we obtain

$$
P_{e_{k}}=P\left(x_{k}(0)+s \leq \frac{\left(x_{e}-t_{c} v_{1}\right)\left(v_{2}-v_{1}\right)}{v_{2}}\right) .
$$

Assuming that the above two conditions are mutually independent, the probability $P_{k}$ of overtaking $k$ vehicles and then successfully taking the exit is $P_{k}=P_{i_{k}} P_{e_{k}}$. In addition, the elapsed time $t_{e_{k}}$ until MyVehicle reaches the exit after overtaking $k$ vehicles is given by

$$
t_{e_{k}}=\left(x_{e}-\mu_{k}-s\right) / v_{1} \text {. }
$$

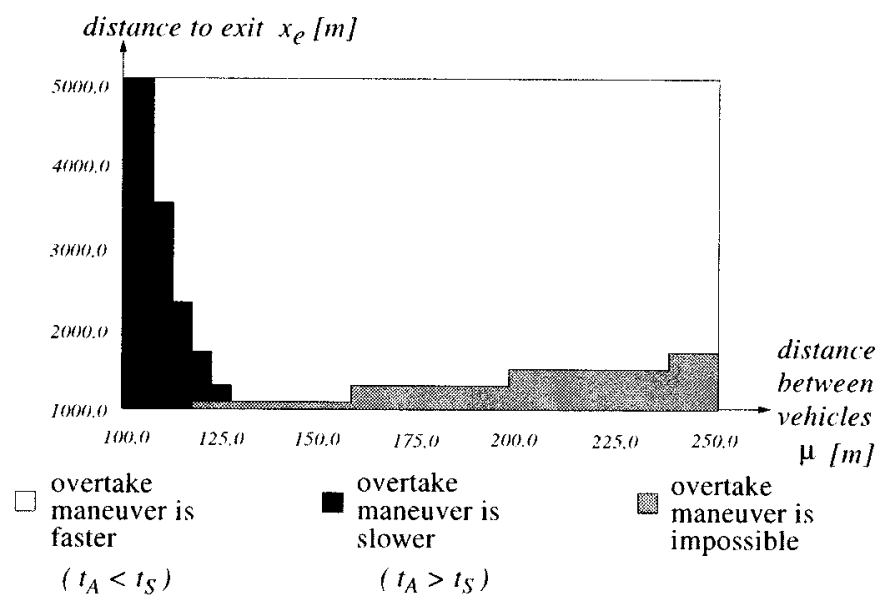

Fig. 10. Comparison of expected arrival times for overtake and keep-lane maneuvers. Gray area indicates the case where overtake maneuver is impossible because the distance to the target exit is too small compared with that to the front vehicle.

Using the above equations, the expectation of the arrival time $t_{A}$ (see Section III-B) is given by

$$
\begin{aligned}
t_{e} & =T_{n}, \\
T_{k} & =P_{k} t_{e_{k}}+\left(1-P_{k}\right) T_{e_{k-1}}, \quad(k=1, \ldots, n) \\
T_{0} & =t_{f}
\end{aligned}
$$

where $n$ is the index of the farthest vehicle that MyVehicle possibly overtakes (i.e., $P_{i_{n}}>0$ and $P_{i_{n+1}}=0$ ); $t_{f}$ is the expectation of the arrival time in case that MyVehicle cannot overtake any vehicles ahead, and can be calculated similarly to the case of $t_{A}$.

On the other hand, the estimated time $t_{S}$ of the keep-lane maneuver (see Section III-B) is given by $x_{e} / v_{1}$. To verify the feasibility of the above model, by using the simulator, we calculated and compared $t_{A}$ and $t_{S}$ for several combinations of $\mu \mathrm{s}$ (the mean of distance between vehicles) and $x_{e} \mathrm{~s}$ (the distance to the target exit) in the case where $v_{1}=80[\mathrm{~km} / \mathrm{h}]$ and $v_{2}=100[\mathrm{~km} / \mathrm{h}]$. The result is summarized in Fig. 10 . From the figure, we see that the larger $\mu$ is, or the larger $x_{e}$ is, the more overtake maneuver has an advantage. This result seems reasonable compared with our intuition.

\section{E. Other Probabilistic Models}

We classified possible situations which may occur in highway driving and, for each situation, we constructed similar probabilistic models to calculate the expected arrival time of each maneuver [15]. In addition to the situation described above, models are constructed for the following situations: overtaking near the target exit without congestion [see Fig. 4(b)], overtaking near an 


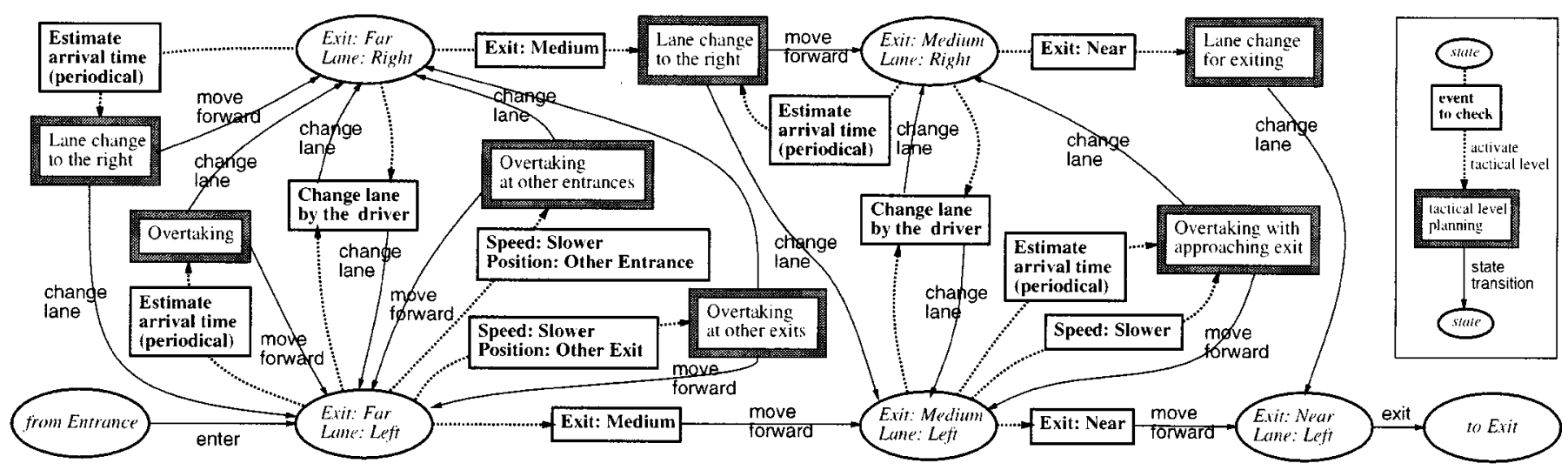

Fig. 11. State-transition graph for metatactical level.

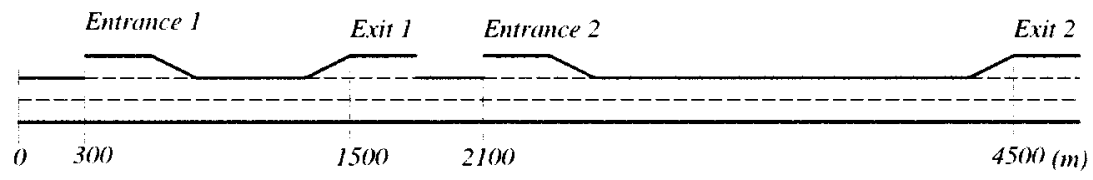

Fig. 12. Highway configuration for simulation.

entrance or an exit which MyVehicle does not take, overtaking without consideration of any entrance or exit, and lane changing for exiting. An appropriate model for the current situation is selected online using the metalevel reasoning described below.

\section{F. Metatactical Level-Reasoning}

The metatactical level (see Fig. 2) continuously watches important events on traffic. Examples of possible events are: the average speed of the current lane slows down; the exit is approaching. It also periodically updates the estimate of the arrival time.

It is inefficient to always check all events. It is also annoying for the driver to be given the same advice such as "keep lane" again and again. Therefore, we need to make the system monitor only selected events which are considered to be important in the current state. To realize such an adaptive focus of attention, we construct a state transition graph shown in Fig. 11. For each state, possible events and their corresponding tactical-level reasoning procedures are retrieved from the graph. For example, at state [Exit: Medium, Lane: Left ] (which means that the distance to the exit is medium and the vehicle is on the left lane), possible events are: 1) the speed becomes slower (Speed: Slower); 2) the estimate of the arrival time is updated (Estimate arrival time); 3 ) the exit becomes near (Exit: Near); and 4) the driver changed the lane without advice (Change lane by the driver). For the first two events, the tactical-level planning overtaking with approaching exit is executed. For the last two events, only the state update is performed. The current transition graph is based on the following assumptions: there are only two lanes and no branches; the right lane is always faster than the left. It is, however, not difficult to extend the graph to remove such assumptions.
TABLE I

Number of Lane Changes (Left) AND Actual Arrival Time in SECONDS (RIGHT)

\begin{tabular}{|c|c|c|c|c|c|c|c|c|}
\hline \multirow[t]{2}{*}{$t_{\text {target }}$} & \multicolumn{8}{|c|}{$C_{\text {change }}$} \\
\hline & \multicolumn{2}{|c|}{100} & \multicolumn{2}{|c|}{400} & \multicolumn{2}{|c|}{900} & \multicolumn{2}{|c|}{1600} \\
\hline 160 & 2.7 & 178.9 & 2.1 & 181.3 & 1.7 & 182.5 & 1.5 & 188.5 \\
\hline 170 & 2.4 & 180.3 & 1.9 & 181 & 1.8 & 186 & 1.5 & 188.5 \\
\hline & 1.9 & 180 & 1.3 & 183 & 0.9 & & 1.0 & 192.9 \\
\hline 190 & 1.7 & 184.5 & 0.9 & 187. & 1.0 & 192.9 & 0.7 & 197.3 \\
\hline 200 & 0.9 & 187.1 & 1.1 & 193.0 & 0.7 & 197.3 & 0.5 & 198.6 \\
\hline
\end{tabular}

\section{G. Simulation Results}

We tested the tactical-level reasoning using the highway traffic simulator. Fig. 12 shows the highway configuration used for simulation. MyVehicle enters at Entrance 1 and exits from Exit 2. The loss function used is

$$
L(t)=\left\{\begin{array}{ll}
0, & t \leq t_{\text {target }} \\
\left(t-t_{\text {target }}\right)^{2}, & t>t_{\text {target }}
\end{array} .\right.
$$

Other vehicles are set to have their own target speed and distance to the front vehicle, and to overtake whenever a slower vehicle is ahead.

We examined the variation of driving of MyVehicle, which is assumed to always follow the generated tactical-level advice, for various target arrival time $t_{\text {target }}$ and the cost of lane changing $C_{\text {change. }}$. For each parameter set, we ran the simulation 15 times with setting the parameters for other vehicles randomly, and calculated the average number of lane changes and the average arrival time. Table I summarizes the result. The earlier the target arrival time is, or the smaller the cost of lane changing is, the more MyVhicle performs lane changing. Since this result coincide with our intuition, we think our tactical-level reasoning is effective. 


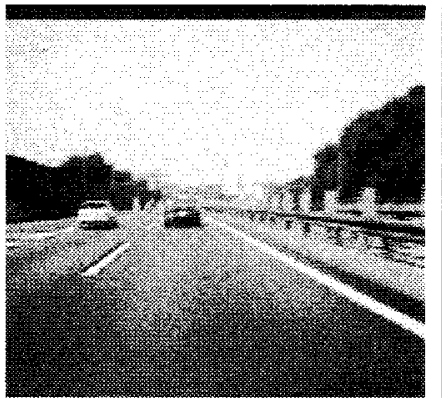

(a)

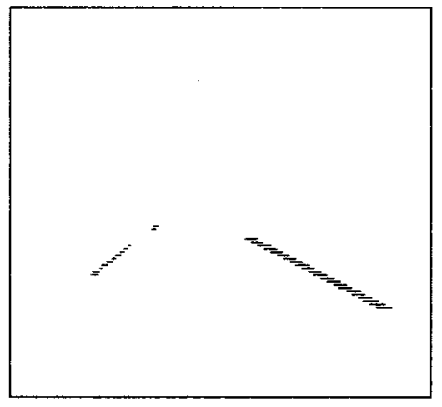

(b)

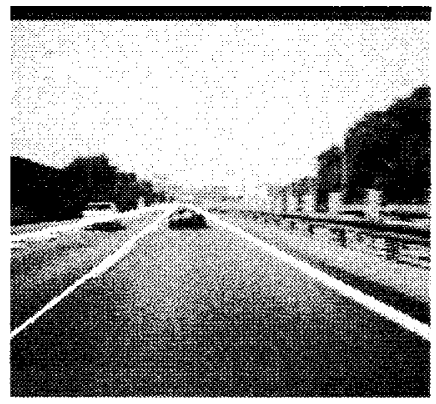

(c)

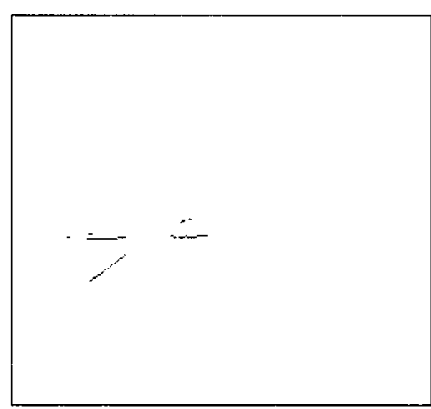

(d)

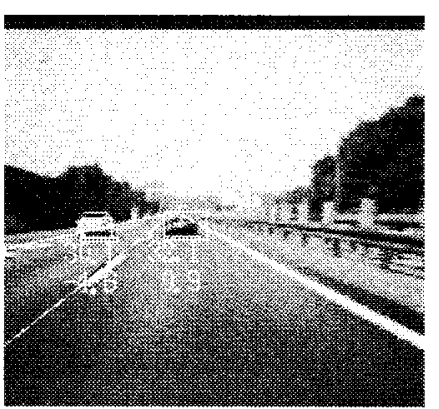

(e)

Fig. 13. Lane and vehicle detection. (a) Input image. (b) Extracted white regions. (c) Detected boundaries. (d) Extracted shadow regions. (e) Detected vehicles and their relative position and velocity.

\section{ROAD SCENE RECOGNITION}

This section describes the road scene recognition subsystem (see Fig. 1) which detects lanes and vehicles. Although many works uses stereo vision (e.g., [16] and [17]), that requires more cameras and more computing power than using a single camera. We, therefore, use a single camera for each direction (front and rear). The rear-directed camera is used for recognizing the vehicles passing from the rear in order to check the safety of lane-changing maneuver. The effective use of the rear camera will be shown in Section V-B. We use shadows under vehicles as the cue for vehicle detection [18].

For the purpose of driver assistance, the recognition system has to process images almost in real-time. On the other hand, we have to consider the uncertainty in visual information for reliable recognition. Therefore, we have decided to adopt a simpler image processing for each frame with an explicit uncertainty modeling, and to statistically integrate information from an image sequence to reduce the uncertainty.

The recognition process is composed of the following steps:

1) Detect lane boundaries and estimate the position of $\mathrm{MyVe}$ hicle;

2) Detect other vehicles and estimates their relative position, relative velocity, and their uncertainties;

3) Make correspondence between frames and integrate data using Kalman filter;

4) Track vehicles based on template matching.

\section{A. Lane Boundary Detection and Vehicle Position Estimation}

First, the system extracts white regions corresponding to the two white boundaries of the current lane by thresholding the image and labeling. Then, a line is fitted to each set of white regions. The region between the two lines is considered as the cur-

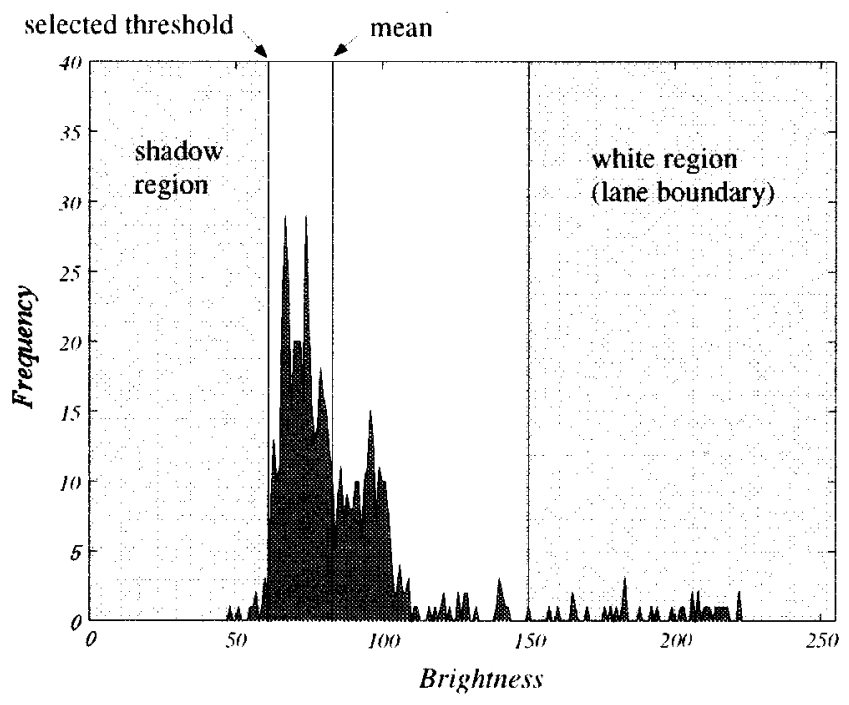

Fig. 14. Example of histogram of the lane region and threshold.

rent lane. Using the width of the lane, the image regions of other lanes can be extracted. Fig. 13(a)-(c) show an input image, the detected white regions, and the detected lane boundaries. From the detected boundaries, the lateral position of MyVehicle (i.e., the lane on which MyVehicle is) is determined. The longitudinal position of MyVhicle is estimated by observing the dashed lane boundaries between lanes. The size and the relative position of such lane boundaries are determined by traffic regulations. By counting how many such boundaries pass MyVehicle, the system can roughly estimate the longitudinal position from the starting point. From the map of the road and this longitudinal position, the system estimates the distance to an entrance or an exit, and if the distance becomes less than a certain threshold, MyVehicle is 


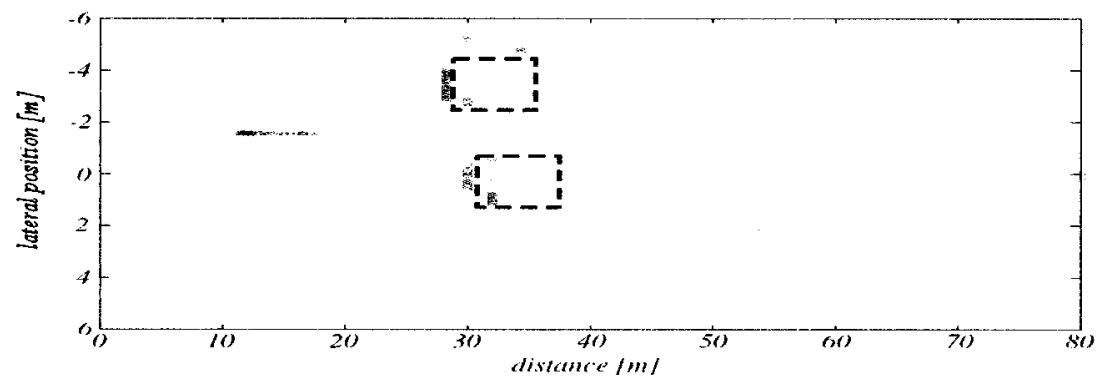

Fig. 15. Projected vehicle position.

considered to reach the entrance or the exit. The empirical accuracy of the longitudinal position was a few hundred meters for a driving of about $12 \mathrm{~km}$. This accuracy will easily be increased by using GPS systems.

\section{B. Vehicle Detection}

Once the lane regions are extracted, the system searches them for vehicles. Since there is a shadow area under a vehicle, we extract a dark region whose brightness is less than a threshold, which is adaptively determined by the mean and variance of the histogram derived from brightness on the lane region (see Fig. 14). Fig. 13(d) shows the extracted shadows for the image shown in Fig. 13(a), which are the candidates of the vehicle position.

Fig. 15 is the projection of shadows and the detected vehicles (dotted rectangle) on the road surface. Since the approximate size of vehicles is known, the shadows of different sizes are determined not to be vehicles.

For each vehicle, we calculate the longitudinal position $z_{i}$ and its uncertainty $\sigma_{z_{i}}^{2}$ (see Fig. 16) by

$$
\begin{aligned}
z_{i} & =\frac{f h}{y_{i}} \\
\sigma_{z_{i}}^{2} & =\left(\frac{f h}{y_{i}^{2}}\right)^{2} \quad \sigma_{y}^{2}=\frac{z_{i}^{4}}{(f h)^{2}} \sigma_{y}^{2},
\end{aligned}
$$

where $y_{i}$ is the averaged vertical position of a dark region and $\sigma_{y}^{2}$ is its variance; the uncertainty of the image position is mainly due to the quantization error.

\section{Making Correspondence Over Frames}

We make correspondence of extracted vehicles over frames for reliable recognition. A newly obtained vehicles is matched with a previously detected vehicle if:

1) both are on the same lane, and;

2) difference of positions is within a certain range computed from the previous uncertainty estimate.

The data of a matched vehicles is integrated with the previous data using Kalman filter [19].

\section{Tracking Using Template Matching}

When a vehicle is detected, the corresponding image region of a certain size is registered as a template. Then the vehicle is tracked by template matching based on the normalized correlation, and the result of the tracking is used for the check of lane changing motion of other vehicles. The rectangles in Fig. 13(e)

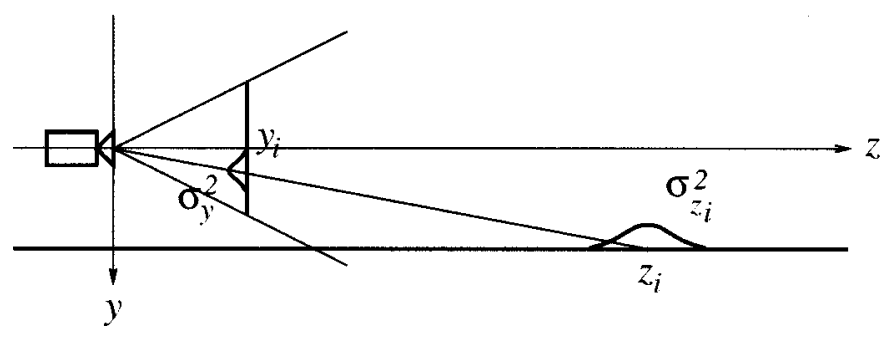

Fig. 16. Calculation of vehicle position and uncertainty.

TABLE II

PROCESSING TIME

\begin{tabular}{l|c}
\hline process & time $(\mathrm{msec})$ \\
\hline \hline lane boundary detection & 4 \\
\hline vehicle detection & 4 \\
\hline filtering & 20 \\
\hline tracking & 17 \\
\hline \hline total & 45 \\
\hline
\end{tabular}

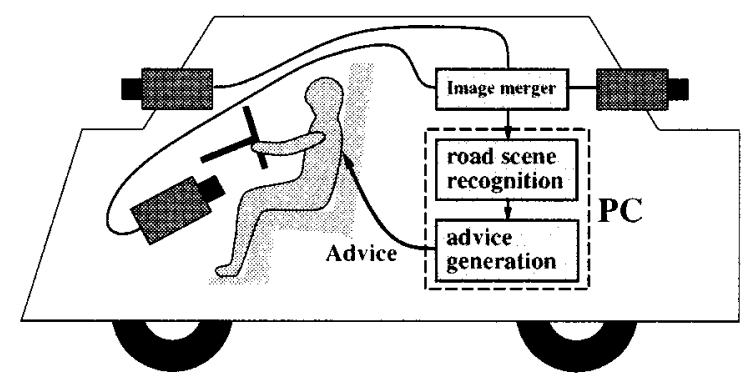

Fig. 17. Configuration of prototype system.

show the results of template matching (each white rectangle indicates a template). The relative position and velocity of each vehicle are also indicated below the template.

\section{E. Processing Time}

Table II shows the computation time for each step of recognition. We used a PentiumII-400 MHz PC with Hitachi IP-5000 image processor and measured the time for the case where three vehicles are being tracked.

\section{EXPERIMENTAL RESULTS}

\section{A. Prototype System and Experiments}

Fig. 17 shows the overview of the prototype system. Two cameras are used to see the forward and the backward directions. 

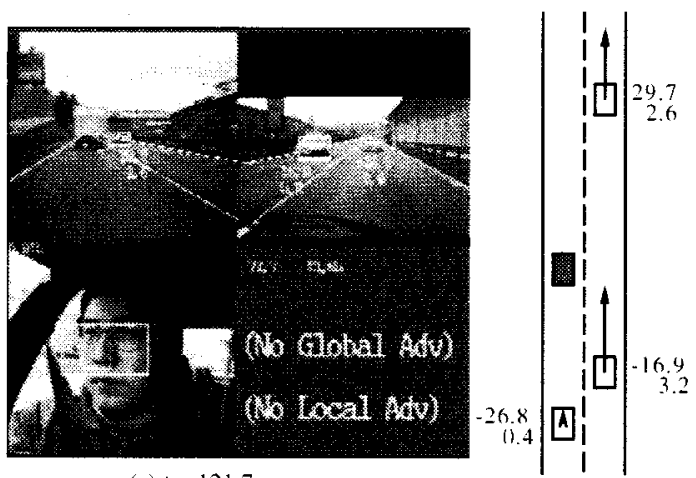

(a) $\mathrm{t}=121.7$
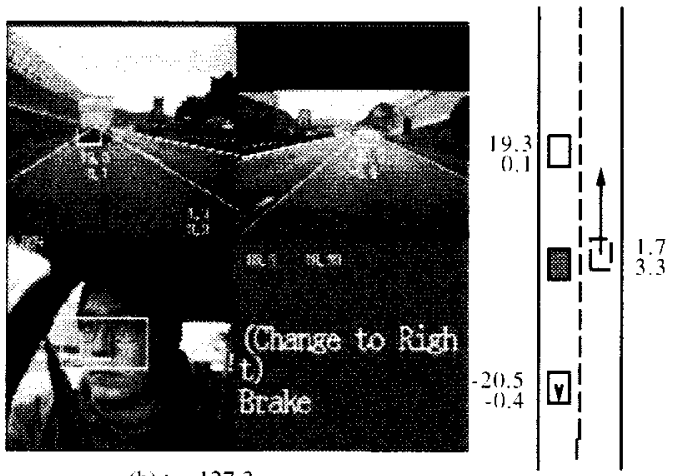

(b) $\mathrm{t}=127.3$
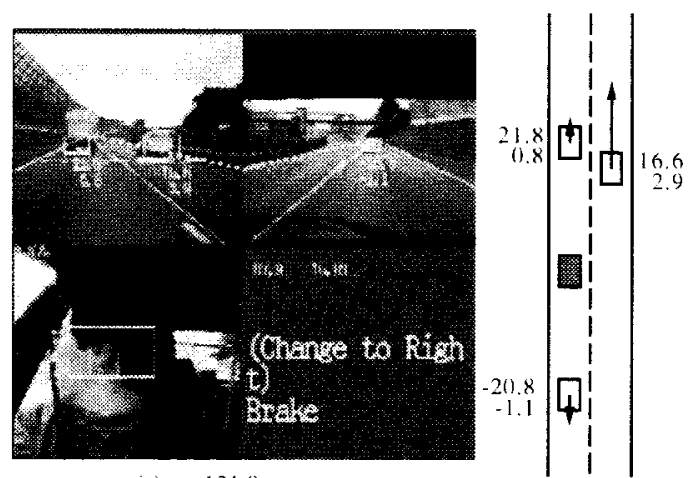

(c) $\mathrm{t}=131.9$
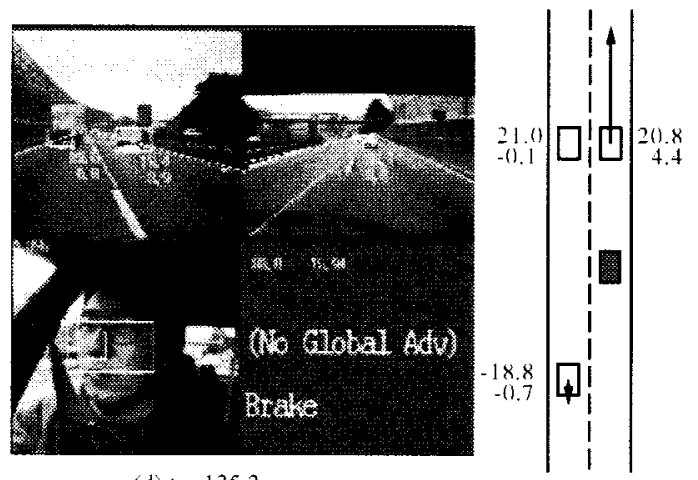

(d) $\mathrm{t}=135.3$

Fig. 18. Driver is advised to change lane later.

Another camera will be used for observing the driver's behavior (e.g., response to the advice); at present, the system detects just the direction of the face, and the information is not used for the assistance. Two subsystems are working on one PC (Pentium-II $400 \mathrm{MHz}$ ), and the processing ability reaches 10 frames/s.

We show the result of an on-road experiment. The vehicle ran from Toyonaka entrance to the Ibaraki exit of the Meishin expressway; the travel distance is about $12 \mathrm{~km}$. We gave the system the loss function indicated by (10), target arrival time $t_{\text {target }}=460 \mathrm{~s}$, and the cost of lane changing $C_{\text {change }}=2500$. This cost means that $50 \mathrm{~s}$ delay is allowed in exchange for lane changing. The actual arrival time was $527 \mathrm{~s}$; during the travel, the system generated the tactical level and the local level advice five times and ten times, respectively.

\section{B. Tactical-Level Advice}

Fig. 18 shows the situation where tactical-level advice "change to right later" was generated due to a reduction of the speed of the current lane. The upper-left and the upper-right part of each image are, respectively, the forward and the backward view. The lower-left part is the observation of the driver, and the advice is displayed on the lower-right part. The corresponding 2-D map of vehicle placement (i.e., relative position and velocity) is also shown at the side of each image. In the map, dotted rectangles indicate vehicles which are invisible but are being tracked by the Kalman filter.

In this case, by referring to the velocity map, only the situation congested was selected. Table III summarizes the measured values of the road scene and the estimated times of arrival at
TABLE III

MeAsured VAlues AND Estimated Times AND Losses

\begin{tabular}{l|l|l}
\hline \multicolumn{2}{l|}{ triggered time $t$} & $126.5(\mathrm{sec})$. \\
\hline \multicolumn{2}{l|}{$t$ distance from the entrance } & $2783(\mathrm{~m})$ \\
\hline \multirow{2}{*}{ average speed } & left & $21.9(\mathrm{~m} / \mathrm{sec})$. \\
\cline { 2 - 3 } & right & $25.3(\mathrm{~m} / \mathrm{sec})$. \\
\hline estimated & keep lane & $421.0(\mathrm{sec})$. \\
\cline { 2 - 3 } arrival time & change lane & $364.3(\mathrm{sec})$. \\
\hline estimated loss & keep lane & 7638.8 \\
\cline { 2 - 3 } & change lane & 3448.6 \\
\hline
\end{tabular}

the goal and their losses. The maneuver "change lane" was selected at the tactical reasoning module, but on the faster lane there was a passing vehicle. The vehicle, which had been detected by the backward camera [see Fig. 18(a)], was tracked by filtering although it was not detected by two cameras at the moment [see Fig. 18(b)]. Thus, the system advised the driver to "change to right later" [see Fig. 18(b)] (note that the advice in the parentheses means that the action should be executed later). Following the advice, the driver checked the faster lane [see Fig. 18(c)], and changed to the lane after the vehicle had passed [see Fig. 18(d)].

\section{Operational-Level Advice}

Fig. 19 shows the situation where a local level advice "brake" was generated due to the deceleration of the front vehicle. At present, this advice is generated if the following condition is satisfied:

$$
d_{\text {curr }}+v_{\text {curr }} T_{f}<d_{\text {thresh }}
$$




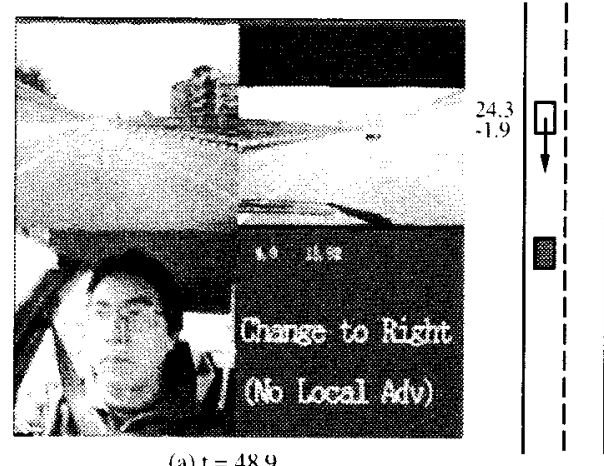

(a) $t=48.9$

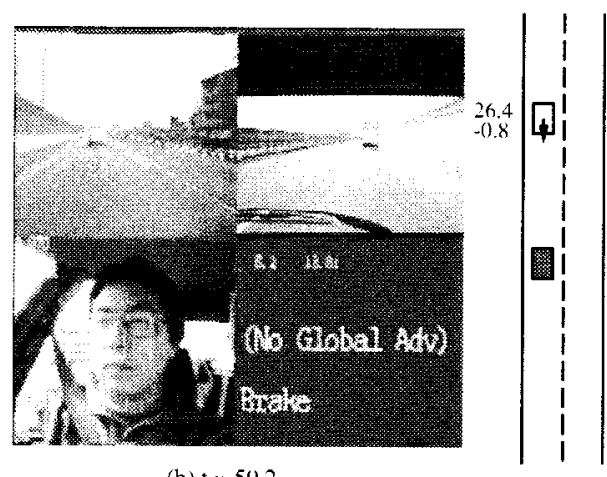

(b) $1=50.2$

Fig. 19. Driver is advised (warned) to brake.

where $d_{\text {curr }}\left(v_{\text {curr }}\right)$ is the current relative position (velocity) of the front vehicle with respect to MyVehicle; $T_{f}$ is a constant time duration [currently $3 \mathrm{~s}$ ]; $d_{\text {thresh }}$ is a threshold [currently $20(\mathrm{~m})]$. In the case of Fig. $19, d_{\text {curr }}$ and $v_{\text {curr }}$ were estimated as $24.5(\mathrm{~m})$ and $-2.0(\mathrm{~m} / \mathrm{s})$; thus, the relative position after $T_{f}$ (s) was estimated as $18.5(\mathrm{~m})$, which is less than $d_{\text {thresh }}$.

\section{CONCLUSION AND Discussion}

This paper has proposed the concept of intelligent navigator that can give the driver timely advice on driving in a dynamic and uncertain traffic environment. The intelligent navigator system is composed of the advice generation and the road scene recognition subsystems. We have proposed a three-level reasoning architecture for tactical-level advance generation. We also constructed a prototype system and conducted experiments on the actual highway. The experimental results show that the intelligent navigator can provide reasonable advice online.

Experimental evaluation of the system like the intelligent navigator is, however, difficult because traffic situations that we would like for evaluation do not necessarily occur at the time of experiments. So we tested and improved our advice-generation strategy by performing simulation repeatedly for various simulated traffic situations, and then, implemented it on the prototype system. We conducted several on-road experiments; during the experiments, one of the authors actually drove the vehicle, and followed the advice when it was generated. His subjective impression is that most of advice were generated in appropriate timings.

It is not an easy task for the driver to give the loss function and the cost assignment, which are used in the tactical-level reasoning. We are planning to employ a learning-based approach in which the parameters are continuously refined through the interaction with the driver.

To deploy the intelligent navigator, the following two issues should be solved. One is the reliability of road-scene recognition. The current vision algorithm works well under good lighting conditions but is not robust against drastic weather changes. We are working on developing more reliable image processing algorithms. Using other kinds of sensors such as millimeter-wave radar [20] may be a practical way. The other issue is the use of (more) global information for increasing the reliability of advice. From the on-vehicle sensors, only the information in the local area can be obtained and more global information is estimated from such local information. For example, we currently estimate the situation of occluded areas indirectly using the speed difference between lanes. It is useful to use roadside cameras [21], if available. In addition, more global information such as the congestion status on a far away location, which will come from various traffic information systems [3], would also enhance the reliability of advice.

\section{REFERENCES}

[1] C. Thorpe, T. Jochem, and D. Pomerleau, "The 1997 automated highway free agent demonstration," in Proc. IEEE Conf. Intelligent Transportation Systems, 1997, pp. 496-501.

[2] D. Özgn̈er et al., "The OSU demo '97 vehicle," in Proc. IEEE Conf. Intelligent Transportation Systems, 1997, pp. 502-507.

[3] Y. Zhao, Vehicle Location and Navigation Systems. Norwood, MA: Artech House, 1997.

[4] J. A. Michon, "A critical view of driver behavior models: What do we know, what should we know?," in Human Behaviors and Traffic Safety, NY: Plenum, 1985.

[5] R. Sukthankar, S. Baluja, J. Hancock, D. Pomerleau, and C. Thorpe, "Adaptive intelligent vehicle modules for tactical driving," in Proc. AAAI Workshop Intelligent Adaptive Agents, 1996, pp. 13-22.

[6] E. Dickmanns and A. Zapp, "A curvature-based scheme for improving road vehicle guidance by computer vision," in Proc. SPIE Conf. Mobile Robots, 1986.

[7] U. Solder and V. Graefe, "Visual detection of distant objects," in Proc. IEEE/RSJ Int. Conf. Intelligent Robots and Systems, 1993, pp. 1042-1049.

[8] C. Stiller, W. Pöchmüller, and B. Hürtgen, "Stereo vision in driver assistance systems," in Proc. IEEE Conf. Intelligent Transportation Systems, 1997, pp. 888-893.

[9] R. Bishop, "A survey of intelligent vehicle applications worldwide," in Proc. IEEE Intelligent Vehicles Symp., 2000, pp. 25-30.

[10] A. Niehaus and R. F. Stengel, "Probability-based decision making for automated highway driving," IEEE Trans. Veh. Technol., vol. 43, pp. 626-634, May 1994.

[11] J. Forbes, T. Huang, K. Kanazawa, and S. Russell, "BATmobile: Toward a Bayesian automated taxi," in Proc. 14th Int. Joint Conf. Artificial Intelligence, 1995, pp. 1878-1885.

[12] E. Gat, "Integrating planning and reacting in a heterogeneous asynchronous architecture for controlling real-world mobile robots," in Proc. AAAI, 1992, pp. 809-815.

[13] B. Pell, D. E. Bernard, S. A. Chien, E. Gat, N. Muscettola, P. P. Nayak, M. D. Wagner, and B. C. Williams, "An autonomous spacecraft agent prototype," in Proc. 1st Int. Conf. Autonomous Agents, Marina del Rey, CA, 1997, pp. 253-261.

[14] E. Yonetani, Transportation Engineering (in Japanese): Kokumin-kagaku-sha, 1977.

[15] M. Itoh, "A driver assistance system based on visual recognition of road scene," (in Japanese), Dept. Computer-Controlled Mechanical Syst., Osaka Univ., Mar. 1999.

[16] Q.-T. Luong, J. Weber, D. Koller, and S. Russell, "An integrated stereobased approach to automatic vehicle guidance," in Proc. 5th Int. Conf. Computer Vision, 1995, pp. 52-57.

[17] C. Knoeppel, A. Schanz, and B. Michaelis, "Robust vehicle detection at large distance using low resolution cameras," in Proc. IEEE Intelligent Vehicles Symp., 2000, pp. 267-272. 
[18] S. Hirata, Y. Shirai, and M. Asada, "Scene interpretation using 3-D information extracted from monocular color images," in Proc. IEEE/RSJ Int. Conf. Intelligent Robots and Systems, 1992, pp. 1603-1610.

[19] N. Ayache and O. D. Faugeras, "Maintaining representations of the environment of a mobile robot," IEEE Trans. Robotics and Automat., vol. RA-5, pp. 804-819, Dec. 1989 .

[20] H. Kuroda, M. Nakamura, K. Takano, and H. Kondoh, "Fully-MMIC $76 \mathrm{GHz}$ radar for ACC," in Proc. IEEE Conf. Intelligent Transportation Systems, 2000, pp. 299-304.

[21] E. Ichihara, H. Takao, and Y. Ohta, "Visual assistance for drivers using roadside cameras," in Proc. IEEE Int. Conf. Intelligent Transportation Systems, 1999, pp. 170-175.

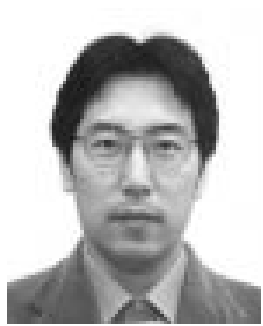

Jun Miura (M'92) received the B.Eng. degree in mechanical engineering and the M.Eng. and the Dr.Eng. degrees in information engineering, all from the University of Tokyo, Tokyo, Japan, in 1984, 1986, and 1989 , respectively.

In 1989, he joined the Department of Computer-Controlled Mechanical Systems, Osaka University, Osaka, Japan, where he is currently an Associate Professor. From March 1994 to February 1995, he was a Visiting Scientist at the Computer Science Department, Carnegie Mellon University, Pittsburgh, PA. His research interests include robotics, artificial intelligence, and computer vision.

Dr. Miura received the Best Paper Award from the Robotics Society of Japan in 1997.

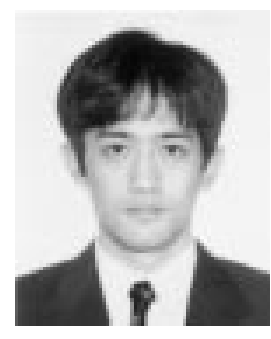

Motokuni Itoh received the B.Eng. and the M.Eng. degrees in computer-controlled mechanical systems from Osaka University, Osaka, Japan, in 1997 and 1999 , respectively. He is currently working toward the Ph.D. degree at the Graduate School of Science and Technology, Kobe University, Kobe, Japan.

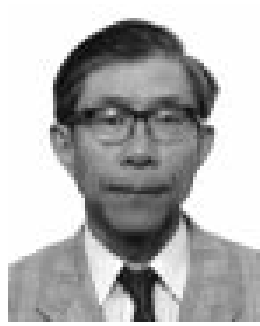

Yoshiaki Shirai received the B.E. degree from Nagoya University in 1964, and the M.E. and Dr.Eng. degrees from the University of Tokyo, Tokyo, Japan, in 1966 and 1969, respectively.

In 1969, he joined the Electrotechnical Laboratory, Tsukuba City, Japan. From 1971 to 1972, he was a Visiting Scholar at the MIT AI Lab. Since 1988, he has been a Professor in the Department of Computer-Controlled Mechanical Systems, Osaka University. His research interests include computer vision, robotics, and artificial intelligence. 